\title{
Mineração
}

\section{Classificação geomecânica em projetos de reforço do teto em mineração de carvão}

\author{
Anderson Luís Weiss \\ Eng.de Minas, Mestre pelo PPGEM, UFRGS.E-mail: anderson.weiss@ufrgs.br \\ André Cezar Zingano \\ Eng.de Minas, Prof. Dr. do DEMIN, UFRGS.E-mail: andrezin@ufrgs.br \\ João Felipe C.L. Costa \\ Eng.de Minas, Prof. Dr. do DEMIN, UFRGS.E-mail: jfelipe@ufrgs.br \\ Jair Carlos Koppe \\ Eng. de Minas/Geól., Prof. Dr. do DEMIN, UFRGS.E-mail: jkoppe@ufrgs.br
}

\begin{abstract}
Resumo
O dimensionamento dos pilares e do reforço do teto em mineração subterrânea de carvão deve levar em consideração parâmetros como o comportamento geomecânico do maciço rochoso e os equipamentos de lavra empregados. Esse artigo demonstra o método de classificação geomecânica baseado no Coal Mine Roof Rating (CMRR), uma ferramenta de engenharia que descreve qualitativamente as condições geológicas e geomecânicas das rochas em minas de carvão para seleção do tipo de suporte de teto. Diferentes índices de CMRR foram identificados e então considerados para definir a largura máxima nos cruzamentos, o comprimento e a capacidade de carregamento dos parafusos de teto. Essa metodologia provou ser adequada, minimizando os riscos de ruptura de teto e predizendo o tipo de suporte mais apropriado a ser empregado às várias zonas dentro do depósito, conforme ilustrado no estudo de caso.
\end{abstract}

Palavras-chave: carvão, geomecânica, lavra subterrânea.

\begin{abstract}
The pillar and roof support design should take into account the rock mass mechanical behavior and excavation method (blasting or mechanical excavation). This work aims to improve the approach for roof support design in underground coal mines to reduce the risks of roof failure and determine the adequate roof support for each kind of roof typologies found in the mine area. This design approach is based on geological mapping and geomechanical classification CMRR - Coal Mine Roof Rating. This engineering tool describes qualitatively the geological and geomechanical conditions of the roof rock mass for coal mines to select appropriate roof support. Different classes of CMRR were identified at Barro Branco coal mine. Therefore, based on the CMRR classification it was considered the maximum roof span at crossings, bolt length and bolt load capacity. The methodology is adequate to reduce roof falls and predicting the adequate support for different roof typologies for different zones in the mining.
\end{abstract}

Keywords: coal, geomechanical classification, underground mining. 


\section{Introdução}

O Estado de Santa Catarina atualmente produz $50 \%$ do carvão nacional. A maioria das minas de carvão de Santa Catarina são subterrâneas e utilizam o método de câmaras-e-pilares. Problemas de suporte e reforço do teto imediato fazem parte da rotina nas fases de planejamento e operação de lavra.

A Mina Barro Branco, pertencente à Indústria Carbonífera Rio Deserto Ltda, minera a camada Bonito. Essa mina está localizada no município de Lauro Müller, situada ao sul do Estado de Santa Catarina.

O teto imediato é formado, basicamente, por arenito laminado ou arenito maciço. De acordo com a qualidade do maciço rochoso do teto, deve ser projetado um tipo de reforço específico.

Atualmente, o tipo de reforço do teto imediato é o mesmo ao longo de toda a mina, independente das variações na tipologia do teto. Acidentes associados a caimentos de rochas são poucos, apenas em situações em que o vão torna-se muito largo, ou quando a ancoragem do parafuso de teto é inadequada ao tipo de teto.

Esse estudo tem como meta averiguar o modelo de reforço de teto empregado atualmente, considerando a qualidade do teto imediato. Esse trabalho aplica uma metodologia para o mapeamento das litologias do teto imediato e sua classificação geomecânica, a qual é baseada a classificação Coal Mine Roof Rating - CMRR (Molinda \& Mark, 1994). Com base nessas informações, será verificado se o padrão de reforço utilizado atualmente é adequado à geometria das escavações e à qualidade do teto imediato.

\section{Metodologia}

A metodologia aplicada nesse trabalho segue as etapas apresentadas a seguir:

(i) Especificar a geologia do teto em cada setor da jazida.

(ii) Determinar a qualidade do teto, com base na classificação CMRR. (iii) Determinar se o reforço aplicado ao teto atualmente é adequado. Se não for adequado, sugerir o reforço com base na classificação CMRR.

Vários autores apresentam metodologias para o dimensionamento do reforço do teto em minas de carvão (Unal, 1983; Peng, 1986; Bieniawski, 1989). A classificação CMRR (Molinda \& Mark, 1994; Mark et al., 2001 e 2002; Weiss, 2003) identifica os fatores litológicos que influenciam na competência estrutural do teto de galerias em minas de carvão e determina seu valor de acordo com o grau de importância de cada camada que forma o teto imediato. A classificação CMRR emprega um sistema de classificação similar à classificação RMR de Bieniawsk (1973 e 1989).

O teto imediato deve ser inicialmente dividido em unidades estruturais. Uma unidade estrutural pode ser designada como uma única camada litológica ou uma seqüência de camadas formando um único intervalo distinto, que deve ter no mínimo 0,15 m de espessura. Em minas de carvão, é comum a presença de finas camadas, que são agrupadas em uma única unidade, devido às semelhanças das propriedades físicas.

Planos de fraqueza como laminação, xistosidade, juntas ou falhas tornam a rocha bastante suscetível à delaminação e ao cisalhamento. Dessa forma, um dos fatores determinantes no cálculo do CMRR é medir a resistência ao cisalhamento desses planos de fraqueza. A capacidade de uma descontinuidade resistir a esse desplacamento é função da coesão e rugosidade das superfícies, as quais devem ser contabilizadas para cada unidade estrutural previamente definida.

A freqüência de descontinuidades de uma família é outro fator que afeta a competência estrutural global da unidade. A freqüência de descontinuidades é determinada pelo inverso do espaçamento entre as descontinuidades (Priest \& Hudson, 1976). Quanto mais próximas elas estiverem dispostas e quanto maior for sua extensão, mais fraco será o maciço rochoso.
Depois de obtido o valor individual, para cada família de descontinuidades presente na unidade estrutural, somando os valores referentes à resistência ao cisalhamento e à freqüência das fraturas, faz-se o ajuste para múltiplas famílias de descontinuidades. Esse ajuste é aplicado quando há mais de um conjunto de falhas na unidade analisada.

A determinação da resistência à compressão das rochas que formam a unidade é necessária na classificação CMRR. Podem ser utilizados testes de carga pontual ou resistência à compressão simples (Mark et al., 2002).

O teste da sensibilidade à umidade é realizado de maneira a observar qual o grau de desagregação da rocha depois de estas estarem imersas em água durante 24 horas. As faces expostas das rochas ou dos caimentos de teto são suscetíveis à infiltração de água e à ação da umidade do ar. Esse processo de intemperismo contribui para a expansão das argilas, favorecendo a delaminação da rocha.

O próximo passo é determinar o grau de importância da unidade estrutural na estabilidade global do teto imediato. A atribuição do peso médio utiliza a espessura da unidade (RRw), que é obtido em função do comprimento do parafuso e o intervalo das unidades em que ele está compreendido.

Outro conceito importante incorporado ao CMRR é o conceito de camada forte. A competência estrutural global das unidades ancoradas pelo parafuso de teto é determinada pela qualidade da camada mais competente no intervalo compreendido pelo parafuso. $\mathrm{O}$ ajuste para camada forte depende de vários fatores. O primeiro é o contraste entre a competência da camada forte em relação às demais unidades. Quanto mais competente for a camada, em relação às demais camadas (unidades), maior será sua contribuição. O segundo fator é a espessura dessa camada, que deve ser, no mínimo, de $0,30 \mathrm{~m}$, para satisfazer a ancoragem e promover qualquer suporte adicional. 
A presença de água nas galerias pode ativar a sensibilidade das rochas do teto, quanto à umidade, e reduzir sua capacidade de suporte. A dedução deste parâmetro é baseada no fluxo de água em toda a sequiência do teto. As observações são realizadas em campo e a quantidade de água que percola pelo teto das galerias é definida ao longo de $3.000 \mathrm{~m}$ de extensão.

A resistência das rochas sobrejacente ao espaço fixado pelos parafusos pode afetar a estabilidade do sistema de suporte. Se as rochas acima do pacote parafusado forem mais fracas, deve-se aplicar uma carga extra sobre o teto. Para isto é empregado um ajuste de sobrecarga.

A conjugação de todos os fatores que contribuem para a competência geomecânica do teto é feita para o cálculo do CMRR.

\section{Estudo de Caso}

A camada de carvão minerada na Mina Barro Branco possui espessura média de $3,47 \mathrm{~m}$, com uma cobertura variando de 20 a 80 metros. O projeto de suporte de teto utilizado na mina baseiase na fórmula de Obert e Durvall (1967), na qual a camada acima do carvão é suspensa com parafusos ancorados no maciço competente. Esse método não considera a qualidade do maciço, apenas considera parâmetros geométricos.

As informações utilizadas nesse trabalho foram reunidas durante uma campanha de amostragem e de observações realizada em campo. O material para os ensaios foi obtido de sondagens, de amostras do teto, utilizando explosivos, e de fragmentos de rocha remanescentes de caimentos.

\subsection{Geologia do teto imediato}

Foram identificadas apenas duas unidades distintas em todo o perímetro da mina: Arenito Laminado e/ou Siltito Arenoso (Unidade 1) e Arenito Maciço (Unidade 2).

Foi construído um mapa de probabilidade de espessura da Unidade 1. Com o objetivo de reproduzir, tanto as características espaciais, quanto as características estatísticas, utilizou-se o método da simulação seqüencial gaussiana (Isaaks, 1990), compilado no programa SGSIM da biblioteca de softwares GSLIB (Deutsch \& Journel, 1998).

Após ser estimada a distribuição das duas unidades presentes na mina, foram mapeadas as estruturas geológicas. Nos trabalhos de campo, observouse a presença de dois conjuntos estruturais (laminação horizontal plano-paralela e falhamentos), dando origem a seis condições geológicas e estruturais distintas, que podem ser encontradas na mina (Figura 1). Na condição 1, é encontrado arenito maciço cortado por fraturas e falhas. Na condição 2 , o arenito maciço não é cortado por descontinuidades. Na condição 3, é encontrado arenito laminado, que é cortado por falhas e fraturas. Na condição 4 , o arenito laminado não é cortado por descontinuida-
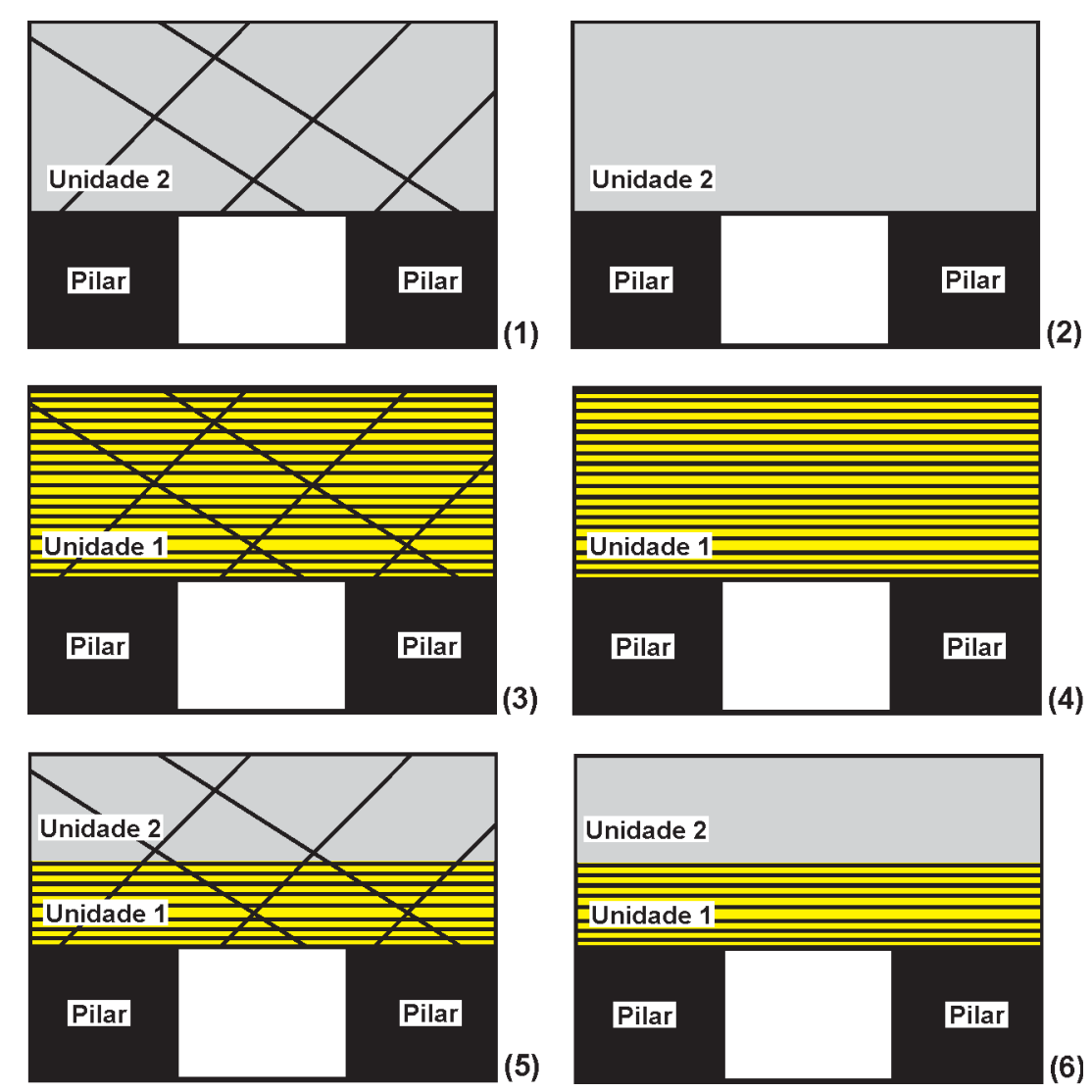

Figura 1 - Cenários de teto imediato na Mina Barro Branco.
(4)

des, existe apenas a laminação do arenito. Nas condições 5 e 6 , o teto imediato é formado por arenito laminado e por arenito maciço mais acima, sendo que, na condição 5 , as duas camadas são cortadas por falhas e fraturas.

As Tabelas 1 e 2 apresentam um resumo das informações para as estruturas geológicas presentes em cada unidade. A Tabela 1 mostra a Unidade 1 com as duas famílias de descontinuidades (laminação e falhas). A Tabela 2 exibe as características geológicas e geomecânicas da estrutura na Unidade 2.

Para cada caso analisado, aplicou-se o ajuste de redução, devido ao desmonte (Kendorki et al., 1983 in Bieniawski, 1989). Esse ajuste relaciona as condições e os métodos de desmonte ao grau de redução a ser aplicado devido ao dano causado nas rochas encaixantes. Os fatores multiplicadores variam de 0,8 , para utilização inadequada de

(6) 
explosivos, a 1,0, para desmonte mecânico. Esse ajuste não pertence à metodologia do CMRR, pois parte do princípio de que o desmonte é realizado mecanicamente, sendo, então, um fator que não interfere na qualidade do maciço rochoso e na estabilidade das escavações subterrâneas. Entretanto as minerações de carvão em subsolo de Santa Catarina operam utilizando explosivos no processo de fragmentação do carvão interferindo na estabilidade do maciço em torno da abertura.

O critério adotado para determinar o fator de ajuste devido ao desmonte tem como base as características estruturais de cada unidade. Quanto melhor for a qualidade estrutural do maciço, menor será a interferência do explosivo na qualidade do teto das galerias.

A Tabela 3 apresenta os valores finais de CMRR, calculados para cada caso. Nos casos 5 e 6, o CMRR foi calculado quando a espessura do arenito laminado é mínima de $0,15 \mathrm{~m}$ (casos $5 \mathrm{~A} \mathrm{e}$ $6 \mathrm{~A})$ e quando o arenito laminado alcança a espessura-limite de 0,75 metros (casos 5B e 6B).

\subsection{Cálculo de reforço do teto imediato}

A largura das galerias é fator importante para manutenção da estabilidade do maciço rochoso que forma o teto imediato. Em minas subterrâneas de carvão, que operam pelo método câmara e pilares, a situação torna-se crítica nos cruzamentos das galerias, onde ocorrem as maiores incidências de caimentos de teto.

O método CMRR estima a distância máxima permitida da diagonal traçada entre dois pontos opostos em uma interseção de galerias $\left(\mathrm{Is}_{\mathrm{G}}\right)$. Para efeitos comparativos, realizaram-se medidas das larguras das diagonais no cruzamento das galerias. A discrepância entre os valores varia de 7,55 a 10,45 m, que representam a média das duas diagonais do cruzamento.

Ao considerar a média dos dados amostrados $(8,89 \mathrm{~m})$, observa-se que a diagonal da interseção das galerias também é elevada em relação a diagonal estimada no projeto. Esse aumento do vão das galerias nos cruzamentos é devido às imperfeições do desmonte e à presença de descontinuidades que cortam a camada de carvão, originando a formação de blocos em forma de cunha, favorecendo o caimento de blocos dos pilares. A comparação entre os valores reais da mina com os resultados sugeridos pelo CMRR $\left(\mathrm{Is}_{\mathrm{G}}\right)$ está na Figura 2.

De acordo com os dados praticados, para o vão que alcançar ou ultrapassar 10,45 m de diagonal, o CMRR mínimo requerido é 55 . Esse valor de CMRR só é atingido nos casos 2 e $6 \mathrm{~A}$. O caso 3 apresenta a situação mais crítica, apresentando o menor valor e, conseqüentemente, a menor distância permitida da diagonal nas interseções das galerias.

A determinação do comprimento dos parafusos é outro parâmetro a ser considerado em projetos de suporte de teto para minas de carvão. Os valores de CMRR obtidos servem como guia para seleção preliminar do comprimento de parafuso, sendo este exclusivamente re-
Tabela 2 - Características geológicas e geomecânicas da Unidade 2.

Unidade 2: Arenito Maciço

\begin{tabular}{|c|c|c|c|}
\hline \multirow{2}{*}{\multicolumn{2}{|c|}{ Unidade 2: Arenito Maciço }} & & \\
\hline & & Condição & CMRR \\
\hline Coesão & Fraca (Falhas) & Caso 1 & 52 \\
\hline Rugosidade & Ondulada (Falhas) & Caso 2 & 81 \\
\hline Espaçamento & $>1,8 \mathrm{~m}$ (Falhas) & Caso 3 & 30 \\
\hline \multirow[t]{2}{*}{ Persistência } & > 9 m (Falhas) & Caso 4 & 41 \\
\hline & \multirow{2}{*}{21 a $55 \mathrm{MPa}$} & Caso 5A & 42 \\
\hline Resistência da rocha & & Caso 5B & 36 \\
\hline Sensibilidade à umidade & Não é sensível & Caso 6A & 71 \\
\hline Água subterrânea & Gotejamento intenso nas falhas & Caso 6B & 56 \\
\hline
\end{tabular}
casos analisados.

\section{Unidade 1: Arenito Laminado}

Coesão

Rugosidade

Espaçamento

Persistência

Resistência da rocha

Sensibilidade à umidade

Água subterrânea

$\begin{array}{ll}\text { Moderada (Laminação) } & \text { Fraca (Falhas) } \\ \text { Planar (Laminação) } & \text { Ondulada (Falhas) } \\ <0,06 \mathrm{~m} \text { (Laminação) } & >1,8 \mathrm{~m} \text { (Falhas) } \\ >9 \mathrm{~m} \text { (Laminação) } & >9 \mathrm{~m} \text { (Falhas) }\end{array}$

21 a $55 \mathrm{MPa}$
Levemente sensível

Gotejamento intenso nas falhas
Tabela 3 - Valores de CMRR para os 
lacionado com o mecanismo de sustentação por efeito viga em coluna total. $\mathrm{O}$ método aplicado incorpora os principais fatores em projetos de suporte de teto: largura nos cruzamentos, espessura de cobertura e a competência estrutural do maciço. Para visualização dos resultados, com base na equação (1), geraram-se gráficos comparativos do comprimento do parafuso $\left(\mathrm{L}_{\mathrm{B}}\right)$ em função da espessura de cobertura $(\mathrm{H})$ e do vão na interseção das galerias para os dois casos extremos de CMRR (Figura 3).

$\mathrm{L}_{\mathrm{B}}=\left(\frac{\mathrm{Is}_{\mathrm{G}}}{13}\right) \log \mathrm{H}\left(\frac{100-\mathrm{CMRR}}{100}\right)^{1,5}$

O caso 2 do CMRR é formado apenas pela Unidade 2 (arenito maciço), o qual apresentam boa competência estrutural e é caracerizado pela ausência de falhas no teto. Nesse caso, o método sugere parafusos com aproximadamente 0,08 m (vão da galeria menor e pouca espessura de cobertura), ou seja, para essa condição o método desconsidera o uso de parafusos de teto.

$\mathrm{O}$ caso 3 do CMRR reúne a pior condição de qualidade do teto. Formado apenas pela Unidade 1, a qualidade dessa categoria é afetada pela presença de falhas. Nesse caso, o método sugere parafusos com até $1,20 \mathrm{~m}$ para situações em que o vão no cruzamento de galerias seja de $10,5 \mathrm{~m}$ e a para espessuras de cobertura superiores a 100 metros.

A função de prover ao maciço rochoso maior resistência requer do parafuso uma certa competência de suportar as solicitações exigidas. A metodologia utilizada para cálculo da capacidade de carga mínima sugerida por unidade de comprimento de parafuso $\left(\right.$ PRSUP $_{\mathrm{G}}$ ) baseia-se na distribuição de tensões, representada pela espessura de cobertura e pela qualidade do maciço rochoso que forma o teto. O PRSUP ${ }_{\mathrm{G}}$ requerido aumenta à medida que o valor do CMRR decresce e aumenta a profundidade da camada (Figura 4 e equação 2).

Quando o CMRR atinge valores elevados (Casos 1, 2, 6A e 6B), a solicitação sobre os parafusos é mínima,

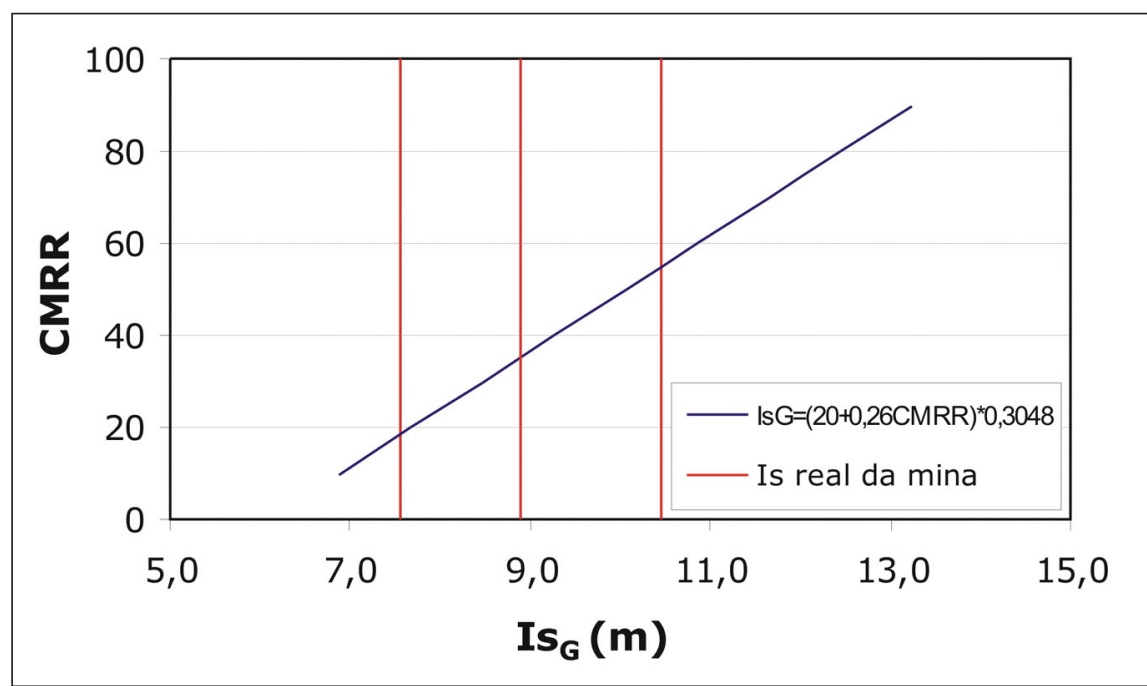

Figura 2 - Largura da diagonal nos cruzamentos das galerias. As linhas verticais representam as larguras $7,55 \mathrm{~m}, 8,89 \mathrm{~m}$ e $10,45 \mathrm{~m}$.

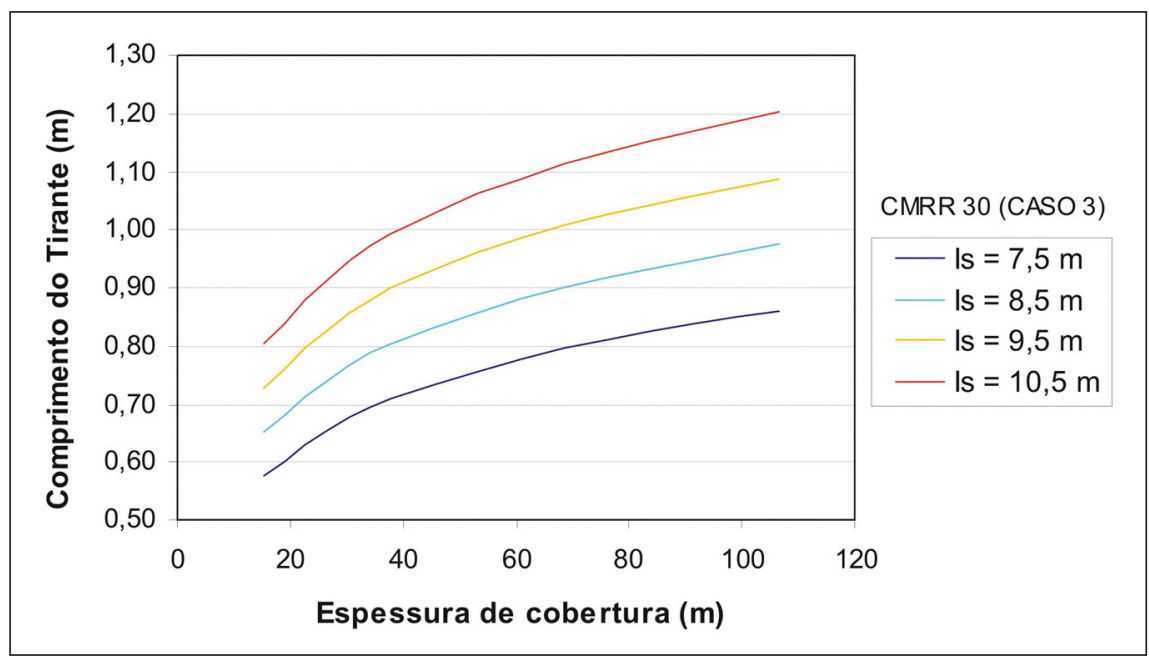

Figura 3 - Comprimento dos parafusos para a pior stuação em diversas larguras de intersecções (Caso 3).

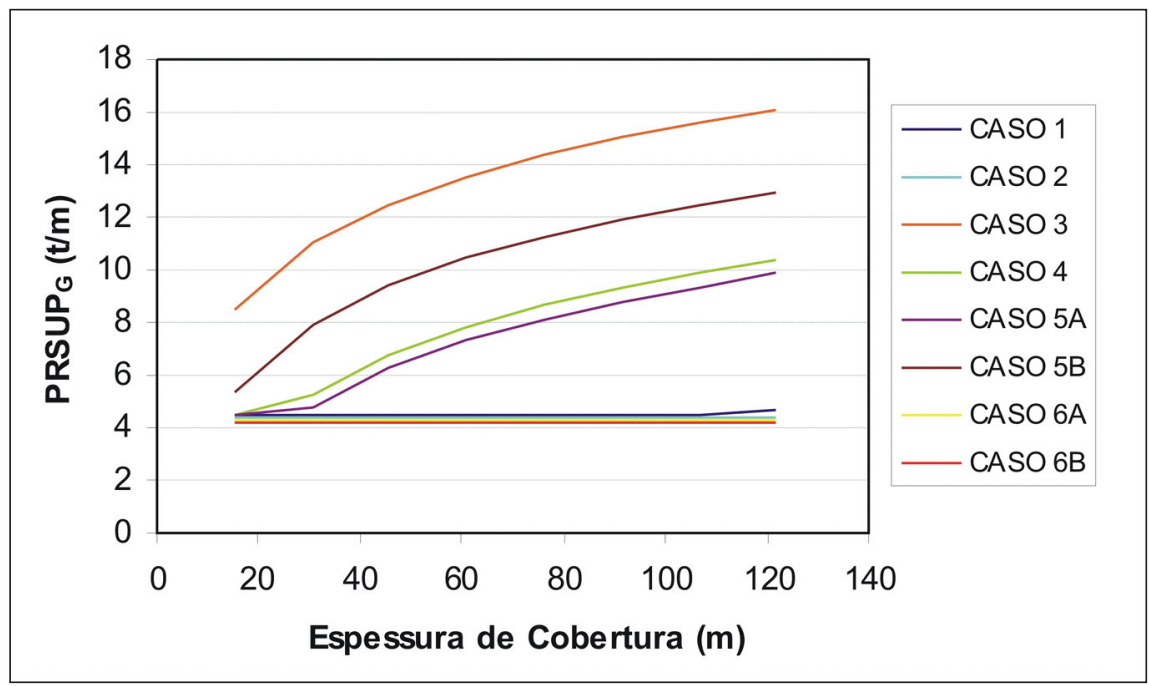

Figura 4 - Capacidade de suporte requerida. 
PRSUP $_{\mathrm{G}}$ de 4,5 t/m. Os casos 4 e $5 \mathrm{~A}$ apresentam uma variação crescente do PR$\mathrm{SUP}_{\mathrm{G}}$ de acordo com a evolução da profundidade da camada. Para uma cobertura de $80 \mathrm{~m}$, o PRSUP ${ }_{\mathrm{G}}$ sugerido é aproximadamente $9 \mathrm{t} / \mathrm{m}$, para as duas situações. A importância da capacidade de suporte do parafuso eleva-se significativamente nos casos 3 e 5B, com valores de $14,5 \mathrm{t} / \mathrm{m}$ e $11,5 \mathrm{t} / \mathrm{m}$, respectivamente.

$\operatorname{PRSUP}_{\mathrm{G}}=(5,7 \log \mathrm{H})-0,35 \mathrm{CMRR}+6,5$

Se comparados com os atuais parafusos da Mina Barro Branco, os quais são instalados pontualmente com resina, essas duas situações avalidas pelo CMRR (Casos 3 e 5B) apresentam-se inadequadas. Testes de arrancamento revelam que a ancoragem resiste em média a 12,7 t de carga. Já a capacidade de carga ou a resistência à tração do parafuso é um pouco inferior, chegando a $12 \mathrm{t}$ para o aço utilizado na sua fabricação.

\section{Conclusões}

A meta de verificar se o padrão de suporte de teto atualmente empregado na Mina Barro Branco apresenta-se adequado às distintas condições geológicas e geomecânicas do maciço rochoso que compõe o teto imediato da mina foi cumprida. A análise determina que o sistema atual de suporte de teto seja modificado.
A classificação geomecânica do teto da Mina Barro Branco, por meio do CMRR, possibilitou mapear as diferentes feições litológicas, as quais interferem na competência do maciço rochoso. As características qualitativas e quantitativas das descontinuidades são os fatores mais importantes na estimativa da qualidade desse maciço. Dessa forma, a aplicação do CMRR demonstrou a existência de seis classes para o teto da mina. Os valores do CMRR ajustado variam de 30 a 81, mostrando a necessidade de cuidados diferenciados para a sustentação do teto. Com base nos índices obtidos, sugere-se uma nova proposta para reforçar o teto imediato, a fim de reduzir os riscos de caimentos em condições adversas e evitar gastos excessivos com suporte em situações favoráveis.

\section{Referências bibliográficas}

ANON. Principais depósitos minerais do Brasil. Textos básicos de geologia e recursos minerais de Santa Catarina. MME, DNPM/Companhia Vale do Rio Doce, v.1, p. 9-17. 1985.

BIENIAWSKI, Z.T. Engineering classification of jointed rock masses. Transactions of South Africa Institute of civil engineers, v.15, p. 335-344, 1973.

BIENIAWSKI, Z.T. Engineering rock mass classification, USA: Jonh Wiley \& Sons, 1989. $251 \mathrm{p}$.

DEUTSCH, C.V., JOURNEL, A.G. GSLIB: Geostatistical Software Library and User's
Guide, 2. ed. New York: Oxford University Press, 1998. 369 p.

ISAAKS, E.H. The application of Monte Carlo Methods to the analysis of spatially correlated data. EUA: Stanford University, 1990. 213 p. (PhD Thesis).

MARK, C., MOLINDA, G.M., DOLINAR, D. Analysis of Roof Bolt Systems (ARBS). 20th International Conference on Ground Control in Mining, West Virginia University. Proceedings... Morgantown, EUA: 2001. p. 218-225.

MARK, C., MOLINDA, G.M. \& BARTON, T. New developments with the coal mine roof rating. 21th International Conference on Ground Control in Mining, West Virginia University. Proceedings... Morgantown, EUA: 2002. p.294-301

MOLINDA, G.M., MARK, C. Coal Mine Roof Rating (CMRR): A Practical Rock Mass Classification for Coal Mines. U.S. Department of the Interior. Pittsburgh, USA: Bureau of Mines, IC 9387, 1994. 83p.

PENG, S.S. Coal mine ground control. New York, USA: John Wiley \& Sons, 1986. 491 p.

PRIEST, S.D., HUDSON, J.A. Discontinuity Spacing in Rock. Int. J. of Rock Mech. Min. Sci. \& Geomech. Abst., Pergamon Press, Inglaterrav.13, p.135-148, 1976.

SALAMON, M.D.G., MUNRO, A.H.A. A Study of the Strength of Coal Pillar. J. S. African Institute of Mining and Metallurgy, Johannesburg, África do Sul, v. 68, p. 5567, 1967.

UNAL, E. Design guidelines and roof control standards for coal mine roofs. USA: The Pennsylvania State University, 1983. 355p. (PhD thesis).

WEISS, A.L. Incorporação de dados geomecânicos em projetos de suporte de teto em mina subterrânea de carvão. PPGEM - UFRGS, 2003. 170 p. (Dissertação de Mestrado).

Artigo recebido em 16/09/2003 e aprovado em 11/06/2004.

\title{
$* * * * * *$
}

\section{REM - Revista Escola de Minas 68 anos divulgando CIÊNCIA.}

$\star \star \star * * *$

Assine e Publique

\author{
$\star * * * * *$
}

